\title{
PANORAMA NACIONAL DAS PESQUISAS SOBRE A GESTÃO DO CONHECIMENTO: UM ESTUDO A PARTIR DA PLATAFORMA SCIELO
}

\author{
Marciano Felipe Sehn (UFPR, Brasil) marciano.sehn@gmail.com \\ Rafael Alexandre dos Reis (UFPR, Brasil) - rafreis2@gmail.com \\ Helena de Fátima Nunes da Silva (UFPR, Brasil) - helenanuness@gmail.com
}

\begin{abstract}
Resumo: A gestão do conhecimento tem conquistado espaço significativo nas pesquisas de diversas áreas de conhecimento, principalmente ao longo dos últimos anos. Apresenta o panorama de pesquisas que envolvem a gestão do conhecimento no Brasil, em relação às suas características e tendências presentes na base de dados SciELO ${ }^{\circledast}$. Realiza pesquisa exploratória, cuja amostra é composta de artigos publicados no Brasil coletados nesta plataforma, cujo termo "Gestão do Conhecimento" encontrava-se presente no resumo. Na pesquisa, as seguintes variáveis foram estudadas: a) as áreas temáticas em que os artigos foram publicados; b) ano de publicação; c) os periódicos em que os artigos são publicados; d) idioma de publicação; e) número de autores; f) palavras chave mais citadas; $g$ ) instituições de ensino segundo filiação dos autores por artigo; h) bibliografia mais utilizada (autores e livros), e, i) artigos de GC relacionados com engenharia. Verificou-se como panorama geral, que os artigos são em maior parte: publicados em periódicos de ciências sociais aplicadas, redigidos no idioma português, publicados a partir de 2007, possuindo de um a três autores. Identificou-se ainda que a bibliografia utilizada é variada, com predominância de autores estrangeiros.
\end{abstract}

Palavras Chave: Gestão do Conhecimento. Panorama. SciELO.

\begin{abstract}
Knowledge management has become increasingly significant in the research of various areas of knowledge, particularly over the past few years. Presents an overview of research involving knowledge management in Brazil, in relation to its characteristics and tendencies present in the database SciELO ${ }^{\circledR}$. Conducts exploratory research, whose sample consists of articles published in Brazil collected in SciELO platform itself, which term "Knowledge Management" was present in the summary. In the survey, the following variables were studied: a) the subject areas in which the articles were published, b) year of publication, c) journal whose articles are published; d) language of publication, e) number of authors; f) keywords most cited g) education institutions second affiliation of authors per article, e, h) most used bibliography (books and authors) and, i) Knowledge Management articles related to engineering. It was found that the papers are mostly: published in journals of applied social sciences, written in Portuguese, published since 2007, having one to three authors. It was also found that the bibliography is varied, with a predominance of foreign authors.
\end{abstract}

Keywords: Knowledge Management. Panorama. SciELO. 


\section{Introdução}

A gestão do conhecimento tem conquistado espaço significativo nas pesquisas de diversas áreas de conhecimento, principalmente ao longo dos últimos anos. Alvarenga Neto (2008) aponta que o conhecimento tem sido reconhecido pelas organizações enquanto ativo intangível, passando a ser contabilizado nos balanços patrimoniais das mesmas, que antes eram restritos a capitais financeiros e físicos.

Tal situação levou a um crescente interesse de pesquisas na área, e uma infinidade de aplicações práticas desses conceitos. Os meios científicos, no entanto, carecem de trabalhos que mostrem o panorama nacional das pesquisas relacionadas à Gestão do Conhecimento no que tange às suas principais características e tendências. Dada esta problemática, a questão de pesquisa do presente artigo é: qual o panorama nacional das pesquisas que envolvem a gestão do conhecimento?

A fim de responder a esta questão, este trabalho tem o objetivo de apresentar o panorama de pesquisas que envolvem a gestão do conhecimento no Brasil, em relação às suas características e tendências, na base de dados SciELO®. Ainda, procura identificar a abordagem dada à GC em trabalhos realizados na área de engenharia dentro do presente contexto.

Sendo os estudos na área de Gestão do Conhecimento relativamente recentes frente a outras ciências, o presente trabalho mostra-se relevante na medida em que possibilita $o$ acompanhamento da evolução e das tendências dessa temática, proporcionando subsídio à tomada de decisão de pesquisadores.

O capítulo a seguir apresenta o referencial teórico desta pesquisa, cobrindo a temática da gestão do conhecimento e da plataforma SciELO®. Em seguida são apresentados os procedimentos metodológicos (capítulo 3), seguido dos resultados no capítulo 4 e das considerações finais no capítulo 5.

\section{Referencial teórico}

O presente capítulo apresenta o marco teórico que fundamentou o desenvolvimento deste artigo. Conceitos sobre Gestão do Conhecimento são apresentados em 2.1, seguidos de teoria sobre a biblioteca eletrônica SciELO® em 2.2 e trabalhos correlatos em 2.3.

\subsection{Gestão do conhecimento}

Para Davenport e Prusak (1998) o conhecimento não é algo novo, no entanto, é novo o reconhecimento deste enquanto ativo corporativo e a compreensão da necessidade de gerencia-lo com os mesmos cuidados que os demais ativos tangíveis da organização.

Assim, tem-se o conhecimento como uma seleção de experiências condensadas, valores, informações contextuais, enfim, toda bagagem que permite a avaliação e incorporação de novas informações e conhecimentos. Nas empresas, de uma maneira geral, esse 
conhecimento é geralmente expresso, tanto na forma de documentos como também, em rotinas, processos normas e práticas organizacionais (DAVENPORT e PRUSAK, 1988).

Segundo Terra (2001) a gestão do conhecimento está ligada a capacidades das empresas em explorar a utilização e combinação de inúmeras fontes de conhecimento organizacional no desenvolvimento de competências específicas e capacidade de inovação, cujos resultados se refletem para a empresa em desenvolvimento de novos produtos, processos, sistemas gerenciais e na liderança de mercado.

De acordo com Nonaka e Takeuchi (2008), conhecimento assume duas formas de expressão: O conhecimento explícito, que é de rápida transmissão aos indivíduos e se apresenta formal e sistematicamente expresso em palavras, números, dados, fórmulas, sons etc. Enquanto que o conhecimento tácito representa o conhecimento que está profundamente firmado nas ações e experiências pessoais dos indivíduos, expressos na forma de ideias, valores e emoções por ele incorporados. Por esse motivo, o conhecimento tácito é tido como altamente pessoal e difícil de formalizar.

O modelo de conversão do conhecimento apresentado por Nonaka e Takeuchi (2008), envolve quatro possibilidades, sendo elas: a socialização, a externalização, a combinação e a internalização. Segundo os autores, o conhecimento é amplificado à medida que passa pelos quatro tipos de conversão, que podem ser descritos da seguinte forma:

Socialização, processo de conversão de conhecimento tácito para tácito, envolve o compartilhamento e criação de conhecimento tácito por meio da experiência direta.

Externalização, processo de conversão do conhecimento tácito para explicito por meio da articulação do conhecimento tácito por meio do diálogo e da reflexão.

Combinação, representa o processo de conversão do conhecimento explicito para explícito, no qual ocorre a sistematização e aplicação do conhecimento explicito e da informação.

Internalização, na qual ocorre a conversão do conhecimento explícito para tácito, etapa na qual ocorre o aprendizado de como se adquire um novo conhecimento tácito na prática.

Cabe ressaltar que, segundo Schlesinger et al (2008), grande parte do conhecimento das organizações não está concentrada em um repositório individual, estando disperso em arquivos, ou sob domínio dos próprios colaboradores, com isso dificultando seu acesso e uso quando desejado. Segundo os autores, essa dificuldade pode ser sanada com a utilização de mapas de conhecimento. De forma análoga, tem-se a plataforma SciELO, cuja função principal é a de possibilitar o acesso e a integridade de trabalhos publicados em uma infinidade de periódicos especializados em diferentes áreas. 
2.2 Scientific Electronic Library Online (SciELO®)

A SciELO - Scientific Electronic Library Online (Biblioteca Científica Eletrônica Online) é uma biblioteca virtual de revistas científicas em formato eletrônico que organiza e publica textos completos de revistas científicas na Internet, bem como é destinada à produção e publicação de indicadores do seu uso e impacto (PACKER et al. 1998).

De acordo com Marcondes e Sayão (2003), entre os componentes da metodologia da SciELO estão: "a publicação eletrônica de edições completas de periódicos científicos e organização de bases de dados de texto completo que podem ser pesquisados e produção de indicadores estatísticos de seu uso e impacto". A biblioteca SciELO inclui também critérios de avaliação de revistas, os quais são baseados nos padrões de comunicação científica internacionais.

O projeto SciELO resultou da parceria entre FAPESP (Fundação de Ciências do Estado de São Paulo) e BIREME (the Latin America and Caribbean Center on Health Sciences Information) bem como instituições nacionais e internacionais relacionadas com a comunicação científica e editores científicos, um projeto piloto foi iniciado com 10 periódicos brasileiros de diferentes áreas de março de 1997 a maio de 1998.

De acordo com Packer et al (1998) a Fapesp tinha como interesse principal nesse projeto, aumentar a visibilidade da produção científica nacional criando mecanismos de avaliação complementares aos do Institute for Scientific
Information (ISI), enquanto que o interesse central da Bireme era o desenvolver uma metodologia para publicação eletrônica que pudesse complementar a metodologia de registro bibliográfico e indexação utilizada na produção descentralizada da base de dados bibliográficos Literatura Latino-Americana e do Caribe em Ciências da Saúde.

De acordo com Marcondes e Sayão (2003), a SciELO é um dos serviços mais importantes prestados pela BIREME para, entre outros, comunidades científicas da América Latina e do Caribe e envolve uma série de metodologias para publicação eletrônica, acesso e preservação do texto completo de revistas por meio da web.

\subsection{Trabalhos correlatos}

Nesta seção são apresentados alguns trabalhos relacionados com a metodologia empregada no desenvolvimento deste artigo, embora os temas abordados pelos trabalhos descritos não tratem, na maioria, do tema Gestão do Conhecimento, eles permitem observar que trabalhos similares já foram realizados em outras áreas, representando uma potencial lacuna para pesquisas.

Santos, Pilatti e Kovaleski (2005), realizam um estudo com o objetivo de quantificar a produção cientifica sobre Ensino de Engenharia de Produção encontrada nas publicações do Encontro Nacional de Engenharia de Produção (ENEGEP) de 2001 a 2004. O trabalho dos autores foi no sentido de traçar um perfil sobre as publicações da área de ensino de engenharia no encontro. 
Luz, Francisco e Santos Jr (2010) num trabalho similar ao anteriormente citado, fazem uma análise dos artigos publicados nos anais do ENEGEP no período de 2005 a 2009, com o objetivo de analisar a produção científica brasileira em relação ao tema Gestão da Inovação. Os autores analisaram 89 artigos em relação aos seguintes critérios: número de autores por artigo; principais autores (filiação, titulação e freqüência de publicação); instituições de ensino segundo filiação dos autores; porcentagem de publicações por estado; bibliografia utilizada (tipo de bibliografia utilizada, livros mais citados, autores mais citados) e metodologia empregada (aplicação, análise, revisão).

Já Gatti Jr (2011) busca traçar um panorama sobre os estudos ligados à gestão do Conhecimento em escolas de gestão de negócios do país visando com isso, identificar a contribuição da universidade ao tópico gestão do conhecimento. A Pesquisa exploratória desenvolvida pelo autor utilizou apenas informações disponíveis na internet nos sites das referidas instituições, grupos de pesquisa e bases de dados. Tal pesquisa leva o autor a concluir sobre a existência de perspectivas de crescimento sobre pesquisas relacionadas ao tema que apenas ganhou destaque nas últimas décadas. Sugere ainda que mais pesquisas sejam realizadas e de maneira mais aprofundada dada a importância do tema.

Almeida et al (2012) realizam uma análise em relação as publicações científicas indexadas na biblioteca eletrônica SciELO cujo tema esteja relacionado com a metodologia "Seis Sigma".
A análise realizada compreendeu sete variáveis (área, ano, idioma, país, autores, palavras chave, titulo do artigo), que foram categorizadas e analisadas com a utilização de gráficos estatísticos e nuvens de palavras.

\section{Procedimentos metodológicos}

Este capítulo apresenta as delimitações da presente pesquisa, bem como os procedimentos de definição de amostra.

\subsection{Classificação da pesquisa}

A presente pesquisa se classifica, quanto à forma de abordagem do problema, como qualitativa no que se refere a análise e seleção dos artigos, e quantitativa no que se refere à apresentação dos resultados. Quanto a sua natureza, caracteriza-se como básica.

Do ponto de vista de seus objetivos, a pesquisa se classifica como exploratória, que para Marconi e Lakatos (2010), caracteriza-se como uma investigação empírica com três finalidades: desenvolver hipóteses, aumentar a familiaridade do pesquisador com um ambiente, fato ou fenômeno para a realização de uma pesquisa futura mais precisa ou modificar e clarificar conceitos.

Em relação a seus procedimentos técnicos, a pesquisa se enquadra como bibliográfica, pois sua fonte de dados são artigos já publicados, neste caso na base de dados SciELO®. 


\subsection{Definição da amostra}

Os trabalhos selecionados para a realização deste artigo foram retirados da base de dados eletrônica SciELO®. A existência da expressão "Gestão do Conhecimento" no resumo do artigo foi o critério adotado para filtrar aqueles que estejam ligados à temática do presente trabalho. Este filtro resultou em um conjunto de 80 artigos compreendidos no período de 1999 a 2012. Devido à identificação de um artigo publicado duas vezes na mesma revista, para este foi considerado apenas a sua primeira publicação, restando assim 79 artigos válidos na amostra a serem analisados. $\mathrm{O}$ levantamento foi realizado na data de 19 de Outubro de 2012, portanto, os resultados apresentados do mesmo ano não representam números totalizados, e sim uma prévia até esta data.

Partindo deste critério, os artigos foram então analisados segundo sete variáveis que podem representar o atual panorama das pesquisas na área da Gestão do Conhecimento, sendo elas: áreas temáticas predominantes; anos de publicação; quantidade de artigos por periódico científico; idioma; número de autores por artigo; instituições de ensino segundo filiação dos autores, livros e autores mais citados e palavras chave predominantes.

Quanto aos artigos relacionados com a área de engenharia, tratados no tópico 4.9, o critério de filtro adotado pelos autores foi os artigos cuja expressão "Gestão do Conhecimento" estivessem presentes no título do trabalho e pertencessem a área de engenharias segundo a classificação da base SciELO.

O software Microsoft Excel® foi utilizado como suporte ao levantamento e análise dos dados em questão, no qual foram criados gráficos e tabelas, que são apresentados no capítulo a seguir, como parte dos resultados da presente pesquisa. Para criar a nuvem de palavras chave dos artigos analisadas foi utilizado o recurso para a geração de nuvem de palavras do site Wordle ${ }^{\mathrm{TM}}$ $<$ http://www.wordle.net/>.

\section{Resultados}

$\mathrm{Na}$ busca realizada na plataforma SciELO foram filtrados artigos publicados cuja expressão "Gestão do Conhecimento" estava presente no resumo dos artigos publicados no Brasil. Resultando essa busca na identificação de 79 artigos válidos.

Este capítulo apresenta os principais resultados encontrados referentes às características dos trabalhos componentes da amostra da pesquisa.

\section{1 Áreas temáticas dos artigos}

A Figura 1 ilustra o percentual obtido por cada área temática nos artigos analisados. É possível observar que mais da metade dos artigos (55\%) está categorizado como relacionado à área de ciências sociais aplicadas. 


\section{ReLAMEP}

\section{FIGURA 1 - ÁREAS TEMÁTICAS}
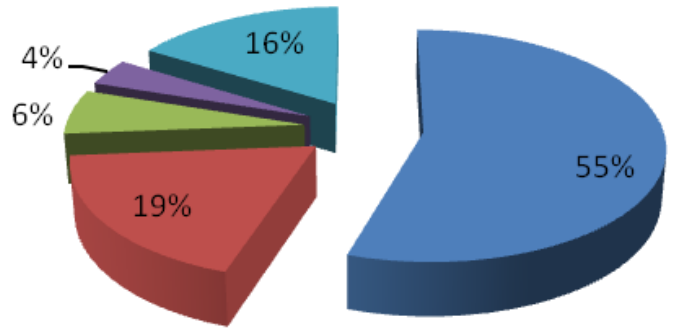

- Ciências Sociais Aplicadas

Engenharia

- Ciências da Saúde

- Ciências Humanas

- Sem área definida

Fonte: Elaborado pelos autores

Adicionalmente nota-se uma quantidade expressiva de artigos na área de engenharia (19\%). Apenas 26\% dos artigos de Gestão do Conhecimento têm como área temática outra que não seja engenharia ou ciências sociais aplicadas, as quais representam $74 \%$ do total.

\subsection{Anos de publicação}

Analisando o número de artigos publicados por ano na figura a seguir (Figura 2), notase um baixo número de trabalhos entre os anos de 1999 a 2006, havendo um aumento significativo no ano de 2007 e obtendo o pico da quantidade em 2009 e 2010. Ressalta-se que o número referente ao ano de 2012 demonstra a quantidade de artigos até o momento do levantamento dos dados (dia 19 de Outubro), não representando, portanto, números totalizados do ano completo.

FIGURA 2 - ANOS DE PUBLICAÇÃO

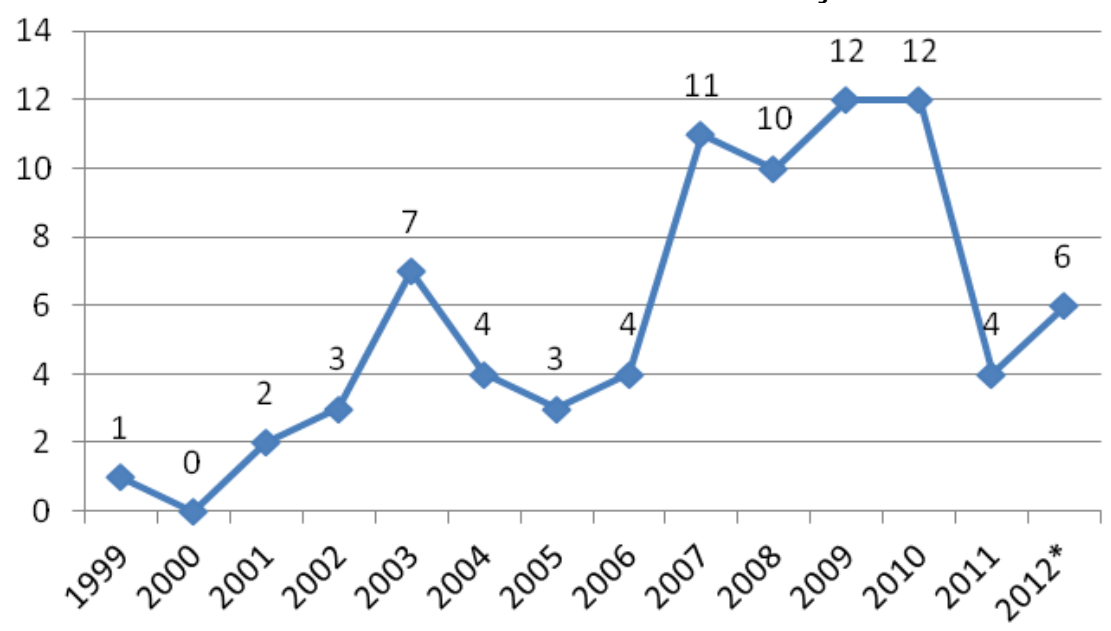

Fonte: Elaborado pelos autores 


\section{ReLAInEP}

\subsection{Quantidade de artigos por periódico científico}

O Quadro 1 mostra o panorama dos artigos no que diz respeito aos periódicos mais publicados. A revista Perspectiva em Ciência da Informação, seguida da revista Ciência da Informação, figuram como as duas com maior quantidade de artigos, representando, juntas, $47 \%$ do total da amostra. Ressalta-se também a presença de periódicos da área de Engenharia de Produção entre os cinco mais publicados. Sendo o caso da revista Gestão e Produção e revista Produção com nove e 6 artigos, respectivamente.

\subsection{Idioma}

Ao se analisar o idioma dos artigos, percebe-se a predominância de artigos em língua portuguesa (95\%), e apenas quatro artigos (5\%) em língua inglesa. A Figura 3 ilustra esta realidade. Tal fato pode ser entendido pelo fato de que a amostra tomada nesta pesquisa contemplou apenas artigos publicados em periódicos brasileiros.

\section{QUADRO 1 - PUBLICAÇÕES POR PERIÓDICO}

\begin{tabular}{|l|c|}
\hline Revistas & Artigos \\
\hline Perspect. ciênc. inf. & 20 \\
\hline Ci. Inf. & 17 \\
\hline Gest. Prod. & 9 \\
\hline JISTEM J.Inf.Syst. Technol. Manag. (Online) & 6 \\
\hline Prod. & 5 \\
\hline Cad. EBAPE.BR & 4 \\
\hline RAE electron. & 3 \\
\hline Rev. Latino-Am. Enfermagem & 3 \\
\hline Estud. av. & 2 \\
\hline Rev. Adm. Pública & 2 \\
\hline Rev. Adm. Contemp. & 2 \\
\hline Pesqui. Oper. & 1 \\
\hline RAM, Rev. Adm. Mackenzie (Online) & 1 \\
\hline REAd. Rev. eletrôn. adm. (Porto Alegre) & 1 \\
\hline Rev. bras. enferm. & 1 \\
\hline Saude soc. & 1 \\
\hline Sociologias & 1 \\
\hline
\end{tabular}

Fonte: Elaborado pelos autores 


\section{ReLAInEP}

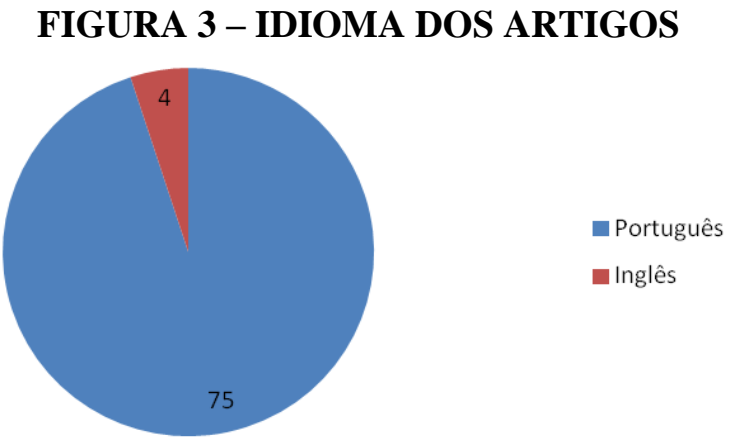

Fonte: Elaborado pelos autores

\subsection{Quantidade de autores por artigo}

Com relação à quantidade de autores em cada um dos artigos, destaca-se que quase a metade destes $(46,8 \%)$ são de autoria de dois autores, seguido pelos de autoria tripla e individual com $21,52 \%$ e $18,99 \%$, respectivamente.

QUADRO 2 - NÚMERO DE AUTORES

\begin{tabular}{|c|c|c|}
\hline $\mathrm{N}^{\text {o de autores }}$ & $\begin{array}{c}\mathrm{N}^{\mathrm{o}} \mathrm{de} \\
\text { artigos }\end{array}$ & $\%$ \\
\hline 1 & 15 & $18,99 \%$ \\
\hline 2 & 37 & $46,84 \%$ \\
\hline 3 & 17 & $21,52 \%$ \\
\hline 4 & 7 & $8,86 \%$ \\
\hline
\end{tabular}

\begin{tabular}{|l|l|l|}
\hline 5 & 3 & $3,80 \%$ \\
\hline
\end{tabular}

Fonte: Elaborado pelos autores

Observa-se também que o número de artigos com cinco autores é muito pouco significativo $(3,8 \%)$. Esta análise demonstra que a maioria dos trabalhos é realizada por um pequeno número de pesquisadores (um, dois ou três).

\subsection{Palavras-chave}

A Figura 4 representa um esquema mostrando as palavras-chave predominantes encontradas nos artigos analisados, sendo que quanto maior o tamanho da fonte de cada palavra, maior sua relevância e predominância da amostra de trabalhos.

Com base na Figura 4, observa-se que as palavras de maior relevância em relação as palavras chave dos artigos que compõem a amostra são: Gestão (1 ${ }^{\circ}$ lugar), Conhecimento $\left(2^{\circ}\right.$ lugar $), \quad$ seguidos de Organizacional e Informação. 


\section{ReLAMEP}

\section{FIGURA 4 - NUVEM DE PALAVRAS-CHAVE}

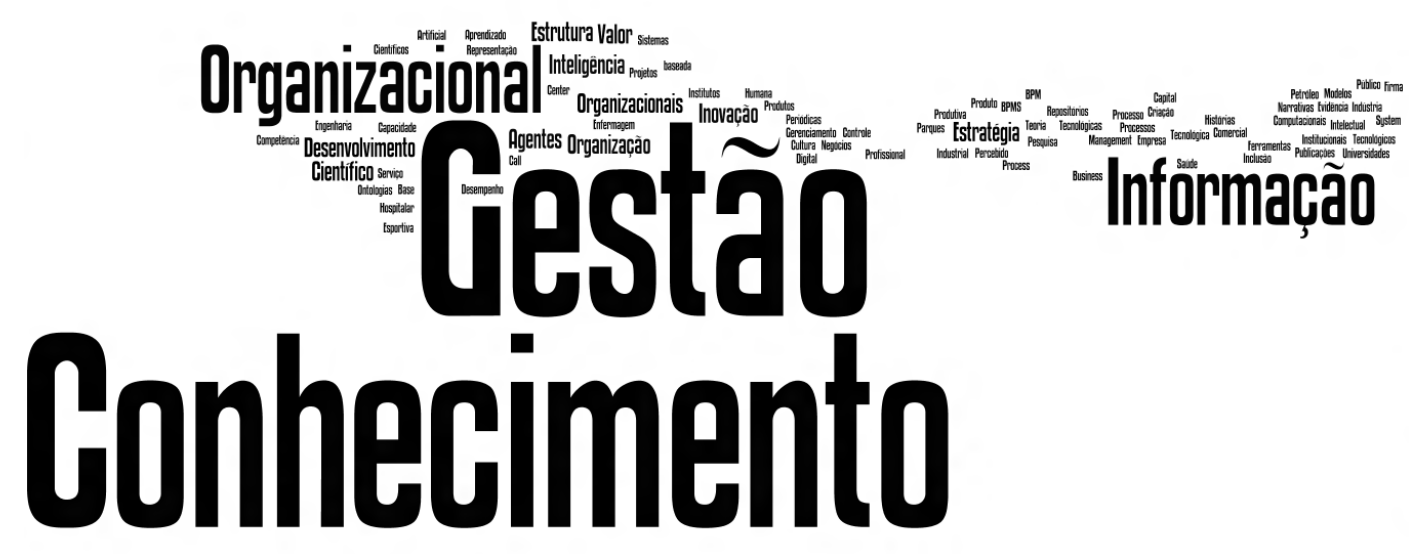

Fonte: Elaborado pelos autores

\subsection{Instituições de ensino segundo filiação dos autores}

A Figura 5 mostra as instituições às quais os autores estão ou estavam afiliados no momento das publicações. Os dados representados na Figura 5 apontam a Universidade Federal de Santa Catarina como a instituição com maior incidência de trabalhos publicados na área da Gestão do Conhecimento (dezoito). Sendo esta seguida da Universidade de São Paulo com treze e da Universidade Federal de São Carlos, com seis artigos. Nota-se que as instituições de ensino privadas com maior incidência de trabalhos foram a Pontifícia Universidade Católica do Paraná e a sua respectiva unidade no Rio Grande do Sul, ambas com quatro publicações, mesmo número de artigos de autores filiados a empresas do setor privado.

\subsection{Bibliografia utilizada}

Esta seção apresenta a bibliografia predominantemente utilizada pelos pesquisadores representantes da amostra estudada nesta pesquisa. Dos 79 artigos que compõem a mesma, foram extraídas 2483 referências, representadas por diversificados materiais como periódicos nacionais e internacionais, livros, teses, dissertações, etc. Evidencia-se, com isso, um número médio de 31 referências por artigo. 


\section{ReLAInEP}

FIGURA 5 - INSTITUIÇÕES PESQUISADAS SEGUNDO O NÚMERO DE PUBLICAÇÕES NA SCIELO.

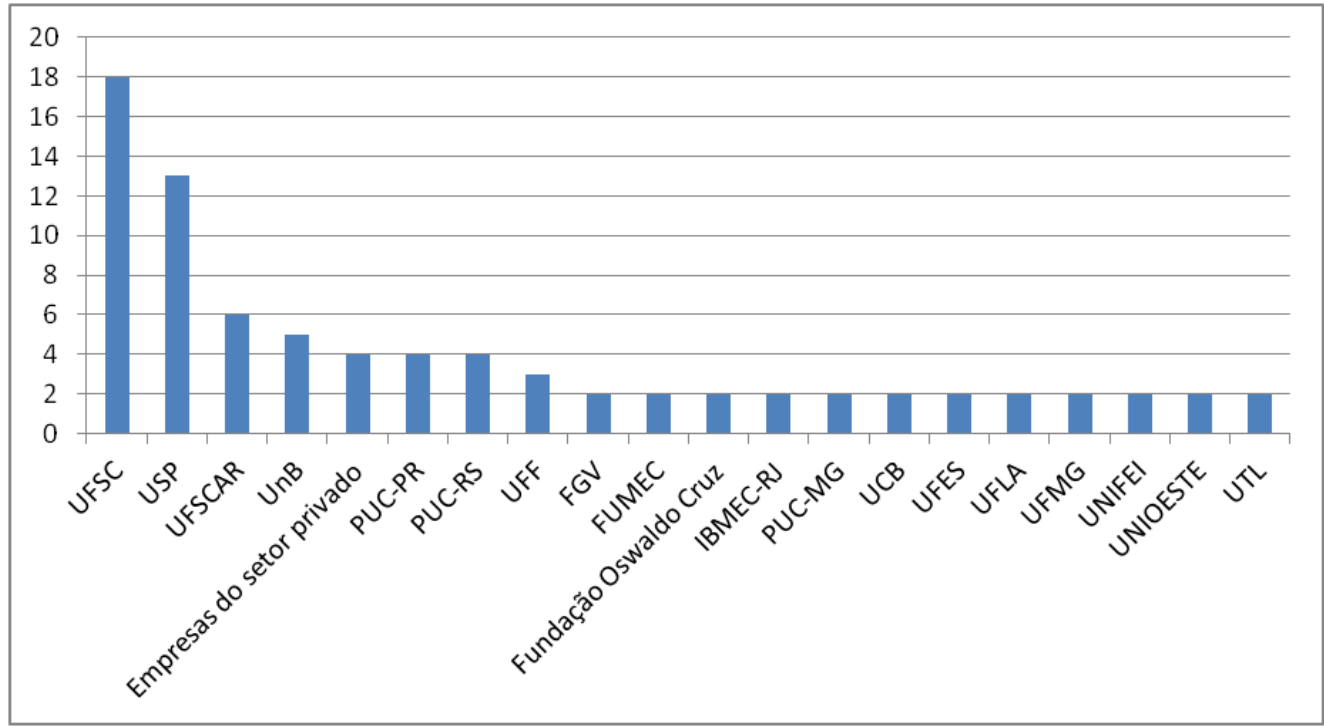

Fonte: Elaborado pelos autores

\subsubsection{Autores}

O Quadro 3 apresenta, em ordem decrescente, os autores com maior número de referências nos artigos analisados.

\section{QUADRO 3 - AUTORES MAIS CITADOS}

\begin{tabular}{|l|c|}
\hline Autor & $\begin{array}{l}\mathrm{N}^{\mathrm{o}} \text { de } \\
\text { Referências }\end{array}$ \\
\hline NONAKA, I.; TAKEUCHI, H. & 55 \\
\hline NONAKA, I. & 38 \\
\hline PRUSAK, L. & 31 \\
\hline STEWART, T. A. & 29 \\
\hline DAVENPORT, T.; PRUSAK, L. & 28 \\
\hline DAVENPORT, T. & 24 \\
\hline TERRA, J. C. C. & 20 \\
\hline CHOO, C. W. & 19 \\
\hline SVEIBY, K. E. & 19 \\
\hline DRUCKER, P. & 18 \\
\hline SENGE, P. M. & 18 \\
\hline SILVA, S. L. & 16 \\
\hline GARVIN, D. A. & 14 \\
\hline WIIG, K. M. & 14 \\
\hline
\end{tabular}

\begin{tabular}{|l|c|}
\hline ARGYRIS, C & 13 \\
\hline POLANYI, M. & 13 \\
\hline YIN, R. K. & 13 \\
\hline PORTER, M. E. & 11 \\
\hline LIEBOWITZ, J. & 10 \\
\hline $\begin{array}{l}\text { EDVINSSON, L.; MALONE, } \\
\text { M. S. }\end{array}$ & 9 \\
\hline FLEURY, A. C. C. & 9 \\
\hline FLEURY, M. T. L. & 9 \\
\hline TEIXEIRA FILHO, J. & 9 \\
\hline
\end{tabular}

Fonte: Elaborado pelos autores

Observa-se a predominância de autores estrangeiros como os mais presentes nas referências dos artigos, sendo materiais publicados por NONAKA e TAKEUCHI, em co-autoria, os mais citados. Materiais publicados unicamente por NONAKA ou em co-autoria com outros autores não presentes na lista aparecem como segundo mais presentes nas referências dos trabalhos. Ressalta-se também a significante relevância de PRUSAK 


\section{ReLAInEP}

e DAVENPORT que, somando-se os trabalhos em co-autoria destes dois ou não, atingem a marca de 83 referências. Observa-se que o autor brasileiro com mais número de referências é TERRA, com um total de vinte.

\subsubsection{Livros}

A partir da análise realizada no item anterior, fez-se o levantamento dos livros com maior número de referências, ilustrado no Quadro 4, a seguir. Em convergência com o apresentado em 4.5.1, o Quadro 4 mostra obras de NONAKA e TAKEUCHI, DAVENPORT e PRUSAK e STEWART como as mais presentes nas referências. Entre as obras de autoria brasileira, destacam-se as de TEIXEIRA FILHO e de TERRA.

QUADRO 4 - LIVROS MAIS CITADOS

\begin{tabular}{|c|c|c|}
\hline Autor & Livro & $\begin{array}{l}\text { Número de } \\
\text { citações }\end{array}$ \\
\hline $\begin{array}{l}\text { NONAKA, I.; } \\
\text { TAKEUCHI, H. }\end{array}$ & $\begin{array}{l}\text { Criação de conhecimento na empresa: como as empresas } \\
\text { japonesas geram a dinâmica da inovação. }\end{array}$ & 53 \\
\hline $\begin{array}{l}\text { DAVENPORT, T.; } \\
\text { PRUSAK, L. }\end{array}$ & $\begin{array}{l}\text { Conhecimento empresarial: como as organizações gerenciam o } \\
\text { seu capital intelectual. }\end{array}$ & 28 \\
\hline STEWART, T. A. & Capital intelectual. & 24 \\
\hline SVEIBY, K. E. & $\begin{array}{l}\text { A nova riqueza das organizações: gerenciando e avaliando } \\
\text { patrimônios do conhecimento. }\end{array}$ & 15 \\
\hline POLANYI, M. & The tacit dimension. & 13 \\
\hline SENGE, P. M. & $\begin{array}{l}\text { A quinta disciplina: arte, teoria e prática da organização de } \\
\text { aprendizagem. }\end{array}$ & 13 \\
\hline YIN, R. K. & Estudo de Caso. & 13 \\
\hline CHOO, C. W. & A organização do conhecimento. & 11 \\
\hline GARVIN, D. A. & Construindo a organização que aprende. & 10 \\
\hline $\begin{array}{l}\text { EDVINSSON, L.; } \\
\text { MALONE, M. S. }\end{array}$ & $\begin{array}{l}\text { Capital intelectual: descobrindo o valor real de sua empresa pela } \\
\text { identificação de seus valores. }\end{array}$ & 9 \\
\hline WILSON, T. D. & The "non-sense" of knowledge management. & 7 \\
\hline $\begin{array}{l}\text { ARGYRIS, C.; } \\
\text { SCHON, D. A. }\end{array}$ & Organizational Learning II: theory, method and practice. & 6 \\
\hline CASTELLS, M. & A sociedade em rede. & 6 \\
\hline $\begin{array}{l}\text { TEIXEIRA FILHO, } \\
\text { J. }\end{array}$ & $\begin{array}{l}\text { Gerenciando conhecimento: como a empresa pode usar a memória } \\
\text { organizacional e a inteligência competitiva no desenvolvimento } \\
\text { de negócios. }\end{array}$ & 6 \\
\hline TERRA, J. C. C. & Gestão do conhecimento: o grande desafio empresarial. & 6 \\
\hline
\end{tabular}

Fonte: Elaborado pelos autores 


\section{ReLAInEP}

4.6 Gestão do Conhecimento na área de Engenharia

O Quadro 5 apresenta uma síntese da contribuição dos referidos artigos à área da engenharia. As informações contidas no quadro foram baseadas nos próprios autores e no conteúdo apresentado por estes.

\section{QUADRO 5 - GESTÃO DO CONHECIMENTO NA ENGENHARIA}

\begin{tabular}{|c|c|c|c|c|}
\hline Ano & Tema & Autores & Objetivo & Principais resultados \\
\hline 1999 & $\begin{array}{l}\text { Tecnologia da } \\
\text { Informação para } \\
\text { Gestão do } \\
\text { Conhecimento em } \\
\text { Organização } \\
\text { Virtual }\end{array}$ & $\begin{array}{l}\text { Luiz Antonio } \\
\text { Joia }\end{array}$ & $\begin{array}{l}\text { Integrar eficazmente diferentes } \\
\text { tecnologias da informação para } \\
\text { aumentar a inteligência de uma } \\
\text { organização virtual responsável } \\
\text { por grandes empreendimentos, } \\
\text { através da criação, implantação, } \\
\text { compartilhamento, armazenamento } \\
\text { e recuperação tanto do } \\
\text { conhecimento tácito como do } \\
\text { explícito. }\end{array}$ & $\begin{array}{l}\text { Apresenta uma modelagem } \\
\text { para integrar diferentes } \\
\text { tecnologias da informação de } \\
\text { modo a aumentar a inteligência } \\
\text { de organizações virtuais. }\end{array}$ \\
\hline 2003 & $\begin{array}{l}\text { Modelo de } \\
\text { avaliação da gestão } \\
\text { do conhecimento } \\
\text { no processo de } \\
\text { desenvolvimento } \\
\text { do produto: } \\
\text { aplicação em um } \\
\text { estudo de caso }\end{array}$ & $\begin{array}{l}\text { Sergio Luis da } \\
\text { Silva, } \\
\text { Henrique } \\
\text { Rozenfeld }\end{array}$ & $\begin{array}{l}\text { Apresentar o modelo de avaliação } \\
\text { e mostrar sua viabilidade prática, } \\
\text { respondendo a seguinte pergunta: } \\
\text { Como este modelo pode ser } \\
\text { aplicado na avaliação prática da } \\
\text { GC em um processo de DP de uma } \\
\text { empresa. }\end{array}$ & $\begin{array}{l}\text { Apresentação de um modelo de } \\
\text { desenvolvimento de produto } \\
\text { baseado na revisão da literatura } \\
\text { e aplicação do modelo em um } \\
\text { estudo de caso, através do qual } \\
\text { identificou-se lacunas para a } \\
\text { melhoria da GC em DP. }\end{array}$ \\
\hline 2004 & $\begin{array}{l}\text { Foresight, } \\
\text { Inteligência } \\
\text { Competitiva e } \\
\text { gestão do } \\
\text { Conhecimento: } \\
\text { Instrumentos para } \\
\text { a Gestão da } \\
\text { Inovação }\end{array}$ & $\begin{array}{l}\text { Claudia } \\
\text { Canongia, } \\
\text { Dalci M. } \\
\text { Santos, } \\
\text { Marcio M. } \\
\text { Santos, } \\
\text { Mauro } \\
\text { Zackiewicz }\end{array}$ & $\begin{array}{l}\text { Busca iniciar um entendimento de } \\
\text { como as sinergias - Foresight, } \\
\text { Inteligência Competitiva e Gestão } \\
\text { do Conhecimento - podem } \\
\text { estimular uma cadeia de agregação } \\
\text { de valor em conhecimento e } \\
\text { influenciar, positivamente, os } \\
\text { processos de tomada de decisão e } \\
\text { de networking. }\end{array}$ & $\begin{array}{l}\text { Discute a aproximação de três } \\
\text { abordagens da gestão da } \\
\text { inovação - Foresight, } \\
\text { Inteligência Competitiva e } \\
\text { Gestão do Conhecimento. }\end{array}$ \\
\hline 2006 & $\begin{array}{l}\text { Gestão do } \\
\text { Conhecimento na } \\
\text { análise de falhas: } \\
\text { mapeamento de } \\
\text { falhas através de } \\
\text { sistema de } \\
\text { informação }\end{array}$ & $\begin{array}{l}\text { Dagoberto } \\
\text { Alves de } \\
\text { Almeida, } \\
\text { Fabiano Leal, } \\
\text { Alexandre } \\
\text { Ferreira de } \\
\text { Pinho, } \\
\text { Liliane Dolores } \\
\text { Fagundes }\end{array}$ & $\begin{array}{l}\text { Apresentar uma metodologia para } \\
\text { o mapeamento do processo de } \\
\text { formação de falhas em } \\
\text { concessionárias de disribuição de } \\
\text { energia elétrica. }\end{array}$ & $\begin{array}{l}\text { Apresenta um modelo de } \\
\text { mapeamento de falhas para } \\
\text { aplicação no setor de } \\
\text { distribuição de energia elétrica. }\end{array}$ \\
\hline 2008 & $\begin{array}{l}\text { Práticas de gestão } \\
\text { do conhecimento } \\
\text { em grupos de } \\
\text { pesquisa da rede } \\
\text { Instituto Fábrica } \\
\text { do Milênio }\end{array}$ & $\begin{array}{l}\text { Karina Kühl de } \\
\text { Lima, } \\
\text { Daniel Capaldo } \\
\text { Amaral }\end{array}$ & $\begin{array}{l}\text { Identificar as práticas de gestão do } \\
\text { conhecimento utilizadas por } \\
\text { grupos de pesquisa que integram a } \\
\text { rede IFM. }\end{array}$ & $\begin{array}{l}\text { Apresenta-se um modelo de } \\
\text { processo de desenvolvimento } \\
\text { de pesquisa, um modelo teórico } \\
\text { de práticas de GC em grupos } \\
\text { de pesquisa, uma lista de } \\
\text { melhores práticas de GC } \\
\text { identificadas e os desafios } \\
\text { enfrentados pelos grupos na } \\
\text { introdução das práticas. }\end{array}$ \\
\hline
\end{tabular}




\begin{tabular}{|c|c|c|c|c|}
\hline 2008 & $\begin{array}{l}\text { Gestão do } \\
\text { conhecimento em } \\
\text { indústria de alta } \\
\text { tecnologia }\end{array}$ & $\begin{array}{l}\text { Isabel Cristina } \\
\text { dos Santos, } \\
\text { João Amato } \\
\text { Neto }\end{array}$ & $\begin{array}{l}\text { Descrever o processo de Gestão do } \\
\text { Conhecimento na indústria } \\
\text { aeronáutica nacional. }\end{array}$ & $\begin{array}{l}\text { Observa que as inovações estão } \\
\text { limitadas aos conteúdos } \\
\text { conhecidos das ciências e suas } \\
\text { aplicações, e são fortemente } \\
\text { orientadas à melhoria contínua, } \\
\text { tendo-se a indústria como } \\
\text { principal agente das mudanças } \\
\text { do setor. }\end{array}$ \\
\hline 2009 & $\begin{array}{l}\text { Um estudo sobre a } \\
\text { adoção de práticas } \\
\text { de gestão do } \\
\text { conhecimento em } \\
\text { organizações } \\
\text { cooperativas }\end{array}$ & $\begin{array}{l}\text { Luciano } \\
\text { Czermainski } \\
\text { Gonçalves, } \\
\text { Edson Pinheiro } \\
\text { de Lima, } \\
\text { Sérgio Eduardo } \\
\text { Gouvêa da } \\
\text { Costa }\end{array}$ & $\begin{array}{l}\text { Determinar o posicionamento de } \\
\text { um conjunto de organizações } \\
\text { cooperativas em relação à adoção } \\
\text { de práticas de gestão do } \\
\text { conhecimento. }\end{array}$ & $\begin{array}{l}\text { Apresenta o desenvolvimento e } \\
\text { o teste de um procedimento de } \\
\text { análise denominado 'Matriz de } \\
\text { Adoção', além dos resultados } \\
\text { de estudo realizado em cinco } \\
\text { unidades cooperativas do } \\
\text { Sudoeste do Paraná, nas quais } \\
\text { identificam-se a presença de } \\
\text { funções e práticas de GC, } \\
\text { embora na maioria delas a } \\
\text { implantação de um modelo de } \\
\text { GC é incompleta. }\end{array}$ \\
\hline 2010 & $\begin{array}{l}\text { Uma Metodologia } \\
\text { para a Gestão no } \\
\text { Processo de } \\
\text { Desenvolvimento } \\
\text { de Produto }\end{array}$ & $\begin{array}{l}\text { Maria } \\
\text { Teresinha } \\
\text { Tamanini } \\
\text { Andrade, } \\
\text { Cristiano } \\
\text { Vasconcelos } \\
\text { Ferreira, } \\
\text { Hernane } \\
\text { Borges de } \\
\text { Barros Pereira } \\
\end{array}$ & $\begin{array}{l}\text { Descrever a elaboração de uma } \\
\text { proposta de Gestão do } \\
\text { Conhecimento utilizando } \\
\text { ontologias, que apoiem a } \\
\text { representação, recuperação e } \\
\text { disseminação do conhecimento } \\
\text { relativo a Processo de } \\
\text { Desenvolvimento de Produto. }\end{array}$ & $\begin{array}{l}\text { Fornece uma dimensão } \\
\text { adicional para classificação e } \\
\text { recuperação do conhecimento, } \\
\text { estabelece um vocabulário } \\
\text { imprescindível ao } \\
\text { compartilhamento e difusão } \\
\text { dos conhecimentos tácitos e } \\
\text { explícitos entre os indivíduos } \\
\text { envolvidos no PDP. }\end{array}$ \\
\hline 2010 & $\begin{array}{l}\text { Laboratório } \\
\text { Nacional de } \\
\text { Astrofísica- um } \\
\text { diagnóstico para } \\
\text { implantação do } \\
\text { programa de } \\
\text { gestão do } \\
\text { conhecimento }\end{array}$ & $\begin{array}{l}\text { Hugo José } \\
\text { Ribeiro Junior, } \\
\text { Rita de Cássia } \\
\text { Magalhães } \\
\text { Trindade Stano }\end{array}$ & $\begin{array}{l}\text { Diagnosticar e verificar a } \\
\text { viabilidade de implantação de um } \\
\text { programa de Gestão do } \\
\text { Conhecimento em uma instituição } \\
\text { de pesquisa e inovação. }\end{array}$ & $\begin{array}{l}\text { Identifica que o LNA possui } \\
\text { um arranjo de grupos com } \\
\text { grandes problemas quanto aos } \\
\text { requisitos da Gestão do } \\
\text { Conhecimento e da } \\
\text { Aprendizagem Organizacional } \\
\text { no que tange os aspectos dos } \\
\text { quatro modos de conversão. }\end{array}$ \\
\hline 2012 & $\begin{array}{l}\text { Gestão do } \\
\text { conhecimento na } \\
\text { manufatura }\end{array}$ & $\begin{array}{l}\text { Heiko Borho, } \\
\text { Alfredo } \\
\text { Iarozinski } \\
\text { Neto, Edson } \\
\text { Pinheiro de } \\
\text { Lima }\end{array}$ & $\begin{array}{l}\text { Identificar os elementos que } \\
\text { caracterizam o inter- } \\
\text { relacionamento entre a estratégia } \\
\text { de manufatura, a estratégia } \\
\text { tecnológica e as práticas de gestão } \\
\text { do conhecimento. }\end{array}$ & $\begin{array}{l}\text { Sintetiza uma discussão acerca } \\
\text { do processo de identificação de } \\
\text { elementos críticos para GC, } \\
\text { propondo um conjunto de } \\
\text { ações de GC observando a } \\
\text { coerência requerida entre as } \\
\text { estratégias de manufatura e } \\
\text { tecnológica. }\end{array}$ \\
\hline 2012 & $\begin{array}{l}\text { Gerenciamento } \\
\text { estratégico da } \\
\text { informação por } \\
\text { meio da utilização } \\
\text { da inteligência } \\
\text { competitiva e da } \\
\text { gestão do } \\
\text { conhecimento }\end{array}$ & $\begin{array}{l}\text { Carlos Eduardo } \\
\text { Roehe } \\
\text { Reginato, } \\
\text { Odacir } \\
\text { Deonísio } \\
\text { Gracioli }\end{array}$ & $\begin{array}{l}\text { Identificar na indústria moveleira } \\
\text { do arranjo produtivo de Bento } \\
\text { Gonçalves -RS como a gestão } \\
\text { estratégica da informação utiliza } \\
\text { seus recursos informacionais e } \\
\text { como estas utilizam a GC e a } \\
\text { inteligência competitiva. }\end{array}$ & $\begin{array}{l}\text { Os resultados alcançados } \\
\text { identificam as características } \\
\text { das empresas quanto à coleta, } \\
\text { difusão e sistematização da } \\
\text { informação e apresentam como } \\
\text { estão sendo utilizadas a gestão } \\
\text { do conhecimento e a } \\
\text { inteligência competitiva. }\end{array}$ \\
\hline
\end{tabular}

Fonte: Elaborado pelos autores (2012) 
A Gestão do Conhecimento pode ser, muitas vezes, erroneamente associada a organizações de base tecnológica e com alto nível de capital intelectual envolvido. A aplicação de princípios desta ciência na engenharia e, sobretudo, na indústria mostra-se plausível quando é feita a análise dos artigos apresentados no quadro acima.

\section{Considerações finais}

A presente pesquisa teve como objetivo apresentar o panorama de pesquisas que envolvem a gestão do conhecimento no Brasil, em relação às suas características e tendências, na base de dados SciELOß.

Observa-se o atendimento ao objetivo proposto, na medida em que características importantes das pesquisas foram evidenciadas, podendo servir como material de subsídio à tomada de decisão de futuros pesquisados no tema da Gestão do Conhecimento.

Pode-se verificar que a maioria dos trabalhos publicados concentra-se em periódicos de ciências sociais aplicadas, foram redigidos em português, publicados a partir de 2007 e têm de um a três autores. A bibliografia utilizada é variada, com predominância de autores estrangeiros. Adicionalmente, instituições de ensino superior públicas estão entre as que mais publicam sobre Gestão do Conhecimento. As pesquisas analisadas no presente artigo têm as Ciências Sociais Aplicadas como área temática predominante, em $55 \%$ das publicações. No entanto, nota-se uma descentralização das áreas temáticas das pesquisas, com uma expressiva presença de artigos na área de Engenharia e Ciências da Saúde, demonstrando a ampla diversidade de campos de aplicação de técnicas e conceitos da Gestão do Conhecimento.

Destacam-se instituições de ensino públicas federais entre as que mais publicam na área de Gestão do Conhecimento, a primeira instituição privada é a Pontifícia Universidade Católica do Paraná (PUC-PR), como quinta colocada.

Adicionalmente, conclui-se que o Brasil ainda carece de uma quantidade maior de autores de referência na área. Entre os dezenove autores mais citados, dois são brasileiros: Sérgio Luís da Silva e José Cláudio Terra, sendo este último o que tem a maior quantidade de referências entre os brasileiros, totalizando 20 citações na amostra de publicações estudada.

Os artigos referentes à Gestão do Conhecimento aplicada a outras áreas buscam, muitas vezes, apontar vantagens obtidas quando da inserção de práticas desta área nas organizações, porém carecem de apresentar a metodogolia de implantação de princípios da Gestão do Conhecimento, discutindo sua viabilidade em termos técnicos e/ou financeiros. Nesse sentido, os trabalhos apresentados por Ribeiro Junior et al (2010), Andrade et al (2010), Santos \& Neto (2008), Almeida et al (2006) e Joia (1999) suprem esta lacuna de conhecimento, no sentido de propor modelos ou apresentar metodologias de implantação da Gestão do Conhecimento em 
diferentes tipos de organizações.

Ainda, a presente pesquisa se mostra de grande importância a pesquisadores e interessados no tema, apresentando um recorte da situação atual de publicações em relação à Gestão do Conhecimento, além disso, a metodologia apresentada pode ser replicada a pesquisas em outras áreas de conhecimento.

Finalmente, como sugestão para trabalhos futuros, aponta-se a ampliação do estudo em outras bases envolvendo também na análise as teses e dissertações, além de uma análise mais aprofundada dos aspectos e características apontados nesta pesquisa.

\section{Referências}

ALMEIDA, C. A.; et al. Seis Sigma: Retrato da Produção Científica Indexada na Biblioteca Eletrônica SCIELO. In: CONGRESSO BRASILEIRO DE ENGENHARIA DE PRODUÇÃO, 2o , 2012. Ponta Grossa. Anais. Ponta Grossa: Associação Paranaense de Engenharia de Produção, 2012.

ALMEIDA, Dagoberto Alves de; LEAL, Fabiano; PINHO, Alexandre Ferreira de and FAGUNDES, Liliane Dolores. Gestão do Conhecimento na análise de falhas: mapeamento de falhas através de sistema de informação. Prod. [online]. 2006, vol.16, n.1, pp. 171-188.

ALVARENGA NETO, R. C. D.. Gestão do Conhecimento em organizações: proposta de mapeamento conceitual integrativo. São Paulo: Saraiva, 2008

ANDRADE, Maria Teresinha Tamanini; FERREIRA, Cristiano Vasconcelos and PEREIRA, Hernane Borges de Barros. Uma ontologia para a Gestão do Conhecimento no Processo de Desenvolvimento de Produto. Gest. Prod. [online]. 2010, vol.17, n.3, pp. 537551.
CANONGIA, Claudia; SANTOS, Dalci M.; SANTOS, Marcio M. and ZACKIEWICZ, Mauro. Foresight, inteligência competitiva e gestão do conhecimento: instrumentos para a gestão da inovação. Gest. Prod. [online]. 2004, vol.11, n.2, pp. 231-238.

DAVENPORT, T. H.; PRUSAK, L.. Conhecimento Empresarial: como as organizações gerenciam o seu capital intelectual. Rio de Janeiro: Campus, 1998.

GATTI JUNIOR, W.. Gestão do conhecimento no Brasil: um breve panorama sobre seu ensino e pesquisa. Revista da Faculdade de Administração e Economia [online]. 2011, V. 2, n. 2. Disponível em: https://www.metodista.br/ revistas/revistasmetodista/index.php/Re FAE/article/view Article/2326.

GONCALVES, Luciano Czermainski; LIMA, Edson Pinheiro de and COSTA, Sérgio Eduardo Gouvêa da. Um estudo sobre a adoção de práticas de gestão do conhecimento em organizações cooperativas. Prod. [online]. 2009, vol.19, n.1, pp. 163-189.

JOIA, Luiz Antonio. Tecnologia da informação para gestão do conhecimento em organização virtual. Prod. [online]. 1999, vol.9, n.2, pp. 05-17.

LUZ, L. M.; FRANCISCO, A. C.; SANTOS JR, G.. Produção Científica Brasileira em Gestão da Inovação: Análise das Publicações do ENEGEP entre 2005 e 2009. In: ENCONTRO NACIONAL DE ENGENHARIA DE PRODUÇÃO, 30, 2010. São Carlos. Anais. São Carlos: Associação Brasileira de Engenharia de Produção, 2010.

LIMA, Karina Kühl de and AMARAL, Daniel Capaldo. Práticas de gestão do conhecimento em grupos de pesquisa da rede Instituto Fábrica do Milênio. Gest. Prod. [online]. 2008, vol.15, n.2, pp. 291305.

MARCONDES, C. H.; SAYÃO, L. F.. The SciELO brazilian scientific journal gateway and Open Archives: a report on the development of the SciELO. Open Archives Data Provider Server. D-Lib Magazine [online]. 2003, v. 6, n. 3. Disponível em: 
<http://www.dlib.org/dlib/march03/ marcondes /03marcondes.html>.

MARCONI, M.A. \& LAKATOS, E.M. Fundamentos de Metodologia Científica. 7. ed. São Paulo: Atlas, 2010.

MENEGHINI, R.. Avaliação da produção científica e o Projeto SciELO. Ciência da Informação [online]. 1998, v. 27, n. 2. Disponível em: http://www.scielo.br/scielo.php?script=sci_art text\&pid=S0100-

19651998000200018\&lng=en\&nrm=iso.

REGINATO, Carlos Eduardo Roehe and GRACIOLI, Odacir Deonísio. Gerenciamento estratégico da informação por meio da utilização da inteligência competitiva e da gestão do conhecimento: um estudo aplicado à indústria moveleira do RS.Gest. Prod. [online]. 2012, vol.19, n.4, pp. 705716.

RIBEIRO JUNIOR, Hugo José and STANO, Rita de Cássia Magalhães Trindade. Laboratório Nacional de Astrofísica do Ministério da Ciência e Tecnologia: um diagnóstico para implantação do programa de gestão do conhecimento. Gest. Prod. [online]. 2010, vol.17, n.1, pp. 111-121.

SANTOS, E. M.; PILATTI, L. A.; KOVALESKI; J. L.. Ensino de Engenharia de Produção: análise da produção científica do ENEGEP. ENCONTRO NACIONAL
DE ENGENHARIA DE PRODUÇÃO, 25․ Porto Alegre, 2005. Anais. Porto Alegre: Associação Brasileira de Engenharia de Produção, 2005.

SCHLESINGER, C. C. B.; et al. Gestão do Conhecimento na Administração Pública. Curitiba: Instituto Municipal de Administração Pública - IMAP, 2008.

SILVA, Sergio Luis da and ROZENFELD, Henrique. Modelo de avaliação da gestão do conhecimento no processo de desenvolvimento do produto: aplicação em um estudo de caso. Prod. [online]. 2003, vol.13, n.2, pp. 6-20.

TAKEUCHI, H.; NONAKA, I.. Gestão do Conhecimento. Porto Alegre: Bookman, 2008.

TERRA, J. C. C.. Gestão do Conhecimento: um grande desafio empresarial. 3.ed. São Paulo: Negócios Editora, 2001.

BORHO, Heiko; IAROZINSKI NETO, Alfredo and LIMA, Edson Pinheiro de. Manufacturing knowledge management. Gest. Prod.[online]. 2012, vol.19, n.2, pp. 247-264.

SANTOS, Isabel Cristina dos and AMATO NETO, João. Gestão do conhecimento em indústria de alta tecnologia. Prod.[online]. 2008, vol.18, n.3, pp. 569-582. 